\title{
MULTIPLE PERIODIC SOLUTIONS FOR A DISCRETE TIME MODEL OF PLANKTON ALLELOPATHY
}

\author{
JIANBAO ZHANG AND HUI FANG
}

Received 19 May 2005; Revised 25 September 2005; Accepted 27 September 2005

We study a discrete time model of the growth of two species of plankton with competitive and allelopathic effects on each other $N_{1}(k+1)=N_{1}(k) \exp \left\{r_{1}(k)-a_{11}(k) N_{1}(k)-\right.$ $\left.a_{12}(k) N_{2}(k)-b_{1}(k) N_{1}(k) N_{2}(k)\right\}, N_{2}(k+1)=N_{2}(k) \exp \left\{r_{2}(k)-a_{21}(k) N_{1}(k)-a_{22}(k)\right.$ $\left.N_{2}(k)-b_{2}(k) N_{1}(k) N_{2}(k)\right\}$. A set of sufficient conditions is obtained for the existence of multiple positive periodic solutions for this model. The approach is based on Mawhin's continuation theorem of coincidence degree theory as well as some a priori estimates. Some new results are obtained.

Copyright (c) 2006 J. Zhang and H. Fang. This is an open access article distributed under the Creative Commons Attribution License, which permits unrestricted use, distribution, and reproduction in any medium, provided the original work is properly cited.

\section{Introduction}

Many researchers have noted that the increased population of one species of phytoplankton might affect the growth of one or several other species by the production of allelopathic toxins or stimulators, influencing bloom, pulses, and seasonal succession. The study of allelopathic interactions in the phytoplanktonic world has become an important subject in aquatic ecology. For detailed studies, we refer to $[1,2,7,9-11,13]$ and references cited therein.

Maynard-Smith [9] and Chattopadhyay [2] proposed the following two species LotkaVolterra competition system, which describes the changes of size and density of phytoplankton:

$$
\begin{aligned}
& \frac{d N_{1}}{d t}=N_{1}\left[r_{1}-a_{11} N_{1}(t)-a_{12} N_{2}(t)-b_{1} N_{1}(t) N_{2}(t)\right], \\
& \frac{d N_{2}}{d t}=N_{1}\left[r_{2}-a_{21} N_{1}(t)-a_{22} N_{2}(t)-b_{2} N_{1}(t) N_{2}(t)\right],
\end{aligned}
$$

where $b_{1}$ and $b_{2}$ are the rates of toxic inhibition of the first species by the second and vice versa, respectively. 
Naturally, more realistic models require the inclusion of the periodic changing of environment (e.g., seasonal effects of weather, food supplies, etc). For such systems, as pointed out by Freedman and $\mathrm{Wu}$ [5] and Kuang [8], it would be of interest to study the existence of periodic solutions. This motivates us to modify system (1.1) to the form

$$
\begin{aligned}
& \frac{d N_{1}}{d t}=N_{1}(t)\left[r_{1}(t)-a_{11}(t) N_{1}(t)-a_{12}(t) N_{2}(t)-b_{1}(t) N_{1}(t) N_{2}(t)\right] \\
& \frac{d N_{2}}{d t}=N_{1}(t)\left[r_{2}(t)-a_{21}(t) N_{1(t)}-a_{22}(t) N_{2}(t)-b_{2}(t) N_{1}(t) N_{2}(t)\right]
\end{aligned}
$$

where $r_{i}(t), a_{i j}(t)>0, b_{i}(t)>0(i, j=1,2)$ are continuous $\omega$-periodic functions.

The main purpose of this paper is to propose a discrete analogue of system (1.2) and to obtain sufficient conditions for the existence of multiple positive periodic solutions by employing the coincidence degree theory. To our knowledge, no work has been done for the existence of multiple positive periodic solutions for this model using this way.

The paper is organized as follows. In Section 2, we propose a discrete analogue of system (1.2). In Section 3, motivated by the recent work of Fan and Wang [4] and Chen [3], we study the existence of multiple positive periodic solutions of the difference equations derived in Section 2.

\section{Discrete analogue of system (1.2)}

Assume that the average growth rates in (1.2) change at equally spaced time intervals and estimates of the population size are made at equally spaced time intervals, then we can incorporate this aspect in (1.2) and obtain the following system:

$$
\begin{aligned}
& \frac{d N_{1}(t)}{d t} \frac{1}{N_{1}(t)}=r_{1}([t])-a_{11}([t]) N_{1}([t])-a_{12}([t]) N_{2}([t])-b_{1}([t]) N_{1}([t]) N_{2}([t]), \\
& \frac{d N_{2}(t)}{d t} \frac{1}{N_{2}(t)}=r_{2}([t])-a_{21}([t]) N_{1}([t])-a_{22}([t]) N_{2}([t])-b_{2}([t]) N_{1}([t]) N_{2}([t]),
\end{aligned}
$$

where $t \neq 0,1,2, \ldots,[t]$ denotes the integer part of $t, t \in(0,+\infty)$.

By a solution of (2.1), we mean a function $x=\left(x_{1}, x_{2}\right)^{T}$, which is defined for $t \in$ $[0,+\infty)$, and possesses the following properties.

(1) $x$ is continuous on $[0,+\infty)$.

(2) The derivatives $d x_{1}(t) / d t, d x_{2}(t) / d t$ exist at each point $t \in[0,+\infty)$ with the possible exception of the points $t \in\{0,1,2, \ldots\}$, where left-sided derivatives exist.

The equations in (2.1) are satisfied on each interval $[k, k+1)$ with $k=0,1,2, \ldots$ For $k \leq t<k+1, k=0,1,2, \ldots$, integrating (2.1) from $k$ to $t$, we obtain

$$
\begin{aligned}
& N_{1}(t)=N_{1}(k) \exp \left\{\left[r_{1}(k)-a_{11}(k) N_{1}(k)-a_{12}(k) N_{2}(k)-b_{1}(k) N_{1}(k) N_{2}(k)\right](t-k)\right\}, \\
& N_{2}(t)=N_{2}(k) \exp \left\{\left[r_{2}(k)-a_{21}(k) N_{1}(k)-a_{22}(k) N_{2}(k)-b_{2}(k) N_{1}(k) N_{2}(k)\right](t-k)\right\} .
\end{aligned}
$$


Letting $t \rightarrow k+1$, we have

$$
\begin{aligned}
& N_{1}(k+1)=N_{1}(k) \exp \left\{r_{1}(k)-a_{11}(k) N_{1}(k)-a_{12}(k) N_{2}(k)-b_{1}(k) N_{1}(k) N_{2}(k)\right\}, \\
& N_{2}(k+1)=N_{2}(k) \exp \left\{r_{2}(k)-a_{21}(k) N_{1}(k)-a_{22}(k) N_{2}(k)-b_{2}(k) N_{1}(k) N_{2}(k)\right\},
\end{aligned}
$$

for $k=0,1,2, \ldots$ Equation (2.3) is a discrete analogue of system (1.2). Notice that the periodicity of parameters of (2.1) is sufficient, but not necessary for the periodicity of coefficients in (2.3).

In system (2.3), we always assume that $r_{i}, a_{i j}>0, b_{i}>0(i, j=1,2)$ are $\omega$-periodic, that is,

$$
r_{i}(k+\omega)=r_{i}(k), \quad b_{i}(k+\omega)=b_{i}(k), \quad a_{i j}(k+\omega)=a_{i j}(k),
$$

for any $k \in Z$ (the set of all integers), $i, j=1,2$, where $\omega$, a fixed positive integer, denotes the prescribed common period of the parameters in (2.3).

\section{Existence of multiple positive periodic solutions}

In this section, in order to obtain the existence of multiple positive periodic solutions of (2.3), we first make the following preparations.

Let $X$ and $Y$ be normed vector spaces. Let $L: \operatorname{Dom} L \subset X \rightarrow Y$ be a linear mapping and $N: X \rightarrow Y$ be a continuous mapping. The mapping $L$ will be called a Fredholm mapping of index zero if $\operatorname{dim} \operatorname{ker} L=\operatorname{codim} \operatorname{Im} L<\infty$ and $\operatorname{Im} L$ is closed in $Z$. If $L$ is a Fredholm mapping of index zero, then there exist continuous projectors $P: X \rightarrow X$ and $Q: Y \rightarrow Y$ such that $\operatorname{Im} P=\operatorname{ker} L$ and $\operatorname{Im} L=\operatorname{ker} Q=\operatorname{Im}(I-Q)$. It follows that $L$ $\operatorname{Dom} L \cap \operatorname{ker} P:(I-P) X \rightarrow \operatorname{Im} L$ is invertible and its inverse is denoted by $K_{p}$. If $\Omega$ is a bounded open subset of $X$, the mapping $N$ is called $L$-compact on $\bar{\Omega}$ if $(Q N)(\bar{\Omega})$ is bounded and $K_{p}(I-Q) N: \bar{\Omega} \rightarrow X$ is compact. Because $\operatorname{Im} Q$ is isomorphic to $\operatorname{ker} L$, there exists an isomorphism $J: \operatorname{Im} Q \rightarrow \operatorname{ker} L$.

For convenience, we introduce Mawhin's continuation theorem as follows.

Lemma 3.1 [6, page 40] (Continuation theorem). Let L be a Fredholm mapping of index zero and let $N: \bar{\Omega} \rightarrow Z$ be L-compact on $\bar{\Omega}$. Suppose

(a) $L x \neq \lambda N x$ for every $x \in \operatorname{dom} L \cap \partial \Omega$ and every $\lambda \in(0,1)$;

(b) $Q N x \neq 0$ for every $x \in \partial \Omega \cap \operatorname{Ker} L$, and Brouwer degree

$$
\operatorname{deg}_{B}(J Q N, \Omega \cap \operatorname{Ker} L, 0) \neq 0 .
$$

Then $L x=N x$ has at least one solution in $\operatorname{dom} L \cap \bar{\Omega}$.

Let $Z, Z^{+}, R, R^{+}$, and $R^{2}$ denote the sets of all integers, nonnegative integers, real numbers, nonnegative real numbers, and two-dimensional Euclidean vector space, respectively. 
4 Periodic solutions for a discrete plankton model

Suppose $\{g(k)\}$ is an $\omega$-periodic $\left(\omega \in Z^{+}\right)$sequence of real numbers defined for $k \in Z$. Throughout this paper, we will use the following notation:

$$
\begin{aligned}
& I_{\omega}=\{0,1, \ldots, \omega-1\}, \quad \bar{g}=\frac{1}{\omega} \sum_{k=0}^{\omega-1} g(k), \quad \bar{R}_{i}=\frac{1}{\omega} \sum_{k=0}^{\omega-1}\left|r_{i}(k)\right|, \\
& \alpha_{i j}=\bar{a}_{j i} \bar{b}_{i}-\bar{a}_{i i} \bar{b}_{j}, \quad \alpha_{i j}^{\prime}=\bar{a}_{j i} \bar{b}_{i}-\bar{a}_{i i} \bar{b}_{j} e^{\bar{R}_{j} \omega}, \quad \alpha_{i j}^{\prime \prime}=\left(\bar{a}_{j i} \bar{b}_{i} e^{\bar{R}_{j} \omega}-\bar{a}_{i i} \bar{b}_{j}\right) e^{\bar{R}_{i} \omega}, \\
& \beta_{i j}=\bar{a}_{i i} \bar{a}_{j j}+\bar{b}_{i} \bar{r}_{j}-\bar{a}_{i j} \bar{a}_{j i}-\bar{b}_{j} \bar{r}_{i} \\
& \beta_{i j}^{\prime}=\bar{a}_{i i} \bar{a}_{j j} e^{\bar{R}_{j} \omega}+\bar{b}_{i} \bar{r}_{j}-\bar{a}_{i j} \bar{a}_{j i} e^{\bar{R}_{i} \omega}-\bar{b}_{j} \bar{r}_{i} e^{\left(\bar{R}_{i}+\bar{R}_{j}\right) \omega} \text {, } \\
& \beta_{i j}^{\prime \prime}=\bar{a}_{i i} \bar{a}_{j j} e^{\bar{R}_{i} \omega}+\bar{b}_{i} \bar{r}_{j} e^{\left(\bar{R}_{i}+\bar{R}_{j}\right) \omega}-\bar{a}_{i j} \bar{a}_{j i} e^{\bar{R}_{j} \omega}-\bar{b}_{j} \bar{r}_{i}, \\
& \gamma_{i j}=\bar{r}_{i} \bar{a}_{j j}-\bar{r}_{j} \bar{a}_{i j}, \quad \gamma_{i j}^{\prime}=\left(\bar{r}_{i} \bar{a}_{j j} e^{\bar{R}_{j} \omega}-\bar{r}_{j} \bar{a}_{i j}\right) e^{\bar{R}_{i} \omega}, \\
& \gamma_{i j}^{\prime \prime}=\bar{r}_{i} \bar{a}_{j j}-\bar{r}_{j} \bar{a}_{i j} e^{\bar{R}_{j} \omega}, \quad i, j=1,2, i \neq j, \\
& N_{1}(\alpha, \beta, \gamma)=\frac{\beta-\sqrt{\beta^{2}-4 \alpha \gamma}}{2 \alpha}, \quad N_{2}(\alpha, \beta, \gamma)=\frac{\beta+\sqrt{\beta^{2}-4 \alpha \gamma}}{2 \alpha} \quad\left(\alpha \neq 0, \beta^{2}-4 \alpha \gamma>0\right) \text {. }
\end{aligned}
$$

Define

$$
l_{2}=\left\{x=\{x(k)\}: x(k) \in R^{2}, k \in Z\right\} .
$$

For $a=\left(a_{1}, a_{2}\right)^{T} \in R^{2}$, define $|a|=\max \left\{\left|a_{1}\right|,\left|a_{2}\right|\right\}$. Let $l^{\omega} \subset l_{2}$ denote the subspace of all $\omega$-periodic sequences equipped with the usual supremum norm $\|\cdot\|$, that is, for $x=$ $\{x(k): k \in Z\} \in l^{\omega},\|x\|=\max _{k \in I_{\omega}}|x(k)|$. It is not difficult to show that $l^{\omega}$ is a finitedimensional Banach space.

Let the linear operator $S: l^{\omega} \rightarrow R^{2}$ be defined by

$$
S(x)=\frac{1}{\omega} \sum_{k=0}^{\omega-1} x(k), \quad x=\{x(k): k \in Z\} \in l^{\omega} .
$$

Then we obtain two subspaces $l_{0}^{\omega}$ and $l_{c}^{\omega}$ of $l^{\omega}$ defined by

$$
\begin{gathered}
l_{0}^{\omega}=\left\{x=\{x(k)\} \in l^{\omega}: S(x)=0\right\}, \\
l_{c}^{\omega}=\left\{x=\{x(k)\} \in l^{\omega}: x(k) \equiv \beta, \text { for some } \beta \in R^{2} \text { and } \forall k \in Z\right\},
\end{gathered}
$$

respectively. Denote by $L: l^{\omega} \rightarrow l^{\omega}$ the difference operator given by $L x=\{(L x)(k)\}$ with

$$
(L x)(k)=x(k+1)-x(k), \quad \text { for } x \in l^{\omega} \text { and } k \in Z .
$$

Let a linear operator $K: l^{\omega} \rightarrow l_{c}^{\omega}$ be defined by $K x=\{(K x)(k)\}$ with

$$
(K x)(k) \equiv S(x), \quad \text { for } x \in l^{\omega} \text { and } k \in Z \text {. }
$$

Then we have the following lemma. 
Lemma 3.2 [12, Lemma 2.1]. (i) Both $l_{0}^{\omega}$ and $l_{c}^{\omega}$ are closed linear subspaces of $l^{\omega}$ and $l^{\omega}=$ $l_{0}^{\omega} \oplus l_{c}^{\omega}, \operatorname{dim} l_{c}^{\omega}=2$.

(ii) $L$ is a bounded linear operator with $\operatorname{ker} L=l_{c}^{\omega}$ and $\operatorname{Im} L=l_{0}^{\omega}$.

(iii) $K$ is a bounded linear operator with $\operatorname{ker}(L+K)=\{0\}$ and $\operatorname{Im}(L+K)=l^{\omega}$.

Lemma 3.3. Let $g, r: Z \rightarrow R$ be $\omega$-periodic, that is, $g(k+\omega)=g(k), r(k+\omega)=r(k)$. Assume that for any $k \in Z$,

$$
g(k+1)-g(k) \leq|r(k)|
$$

Then for any fixed $k_{1}, k_{2} \in I_{\omega}$, and any $k \in Z$, one has

$$
\begin{aligned}
& g(k) \leq g\left(k_{1}\right)+\sum_{s=0}^{\omega-1}|r(s)|, \\
& g(k) \geq g\left(k_{2}\right)-\sum_{s=0}^{\omega-1}|r(s)| .
\end{aligned}
$$

Proof. It is only necessary to prove that the inequalities hold for any $k \in I_{\omega}$. For the first inequality, it is easy to see the first inequality holds if $k=k_{1}$. If $k>k_{1}$, then

$$
g(k)-g\left(k_{1}\right)=\sum_{s=k_{1}}^{k-1}(g(s+1)-g(s)) \leq \sum_{s=k_{1}}^{k-1}|r(s)| \leq \sum_{s=0}^{\omega-1}|r(s)|,
$$

and hence, $g(k) \leq g\left(k_{1}\right)+\sum_{s=0}^{\omega-1}|r(s)|$. If $k<k_{1}$, then $k+\omega>k_{1}$. Therefore,

$$
\begin{aligned}
g(k)-g\left(k_{1}\right) & =g(k+\omega)-g\left(k_{1}\right)=\sum_{s=k_{1}}^{k+\omega-1}(g(s+1)-g(s)) \\
& \leq \sum_{s=k_{1}}^{k+\omega-1}|r(s)| \leq \sum_{s=k_{1}}^{k_{1}+\omega-1}|r(s)|=\sum_{s=0}^{\omega-1}|r(s)|,
\end{aligned}
$$

equivalently, $g(k) \leq g\left(k_{1}\right)+\sum_{s=0}^{\omega-1}|r(s)|$. Now we can claim that the first inequality is valid.

Similar to the above proof, we can prove that the second inequality is valid.

In the following, we make the following assumptions.

$\left(\mathrm{H}_{1}\right) \bar{R}_{i}=(1 / \omega) \sum_{k=0}^{\omega-1}\left|r_{i}(k)\right| \geq(1 / \omega) \sum_{k=0}^{\omega-1} r_{i}(k)>0$.

$\left(\mathrm{H}_{2}\right) \gamma_{i j}^{\prime \prime}=\bar{r}_{i} \bar{a}_{j j}-\bar{r}_{j} \bar{a}_{i j} e^{\bar{R}_{j} \omega}>0, i \neq j, i, j=1,2$.

$\left(\mathrm{H}_{3}\right) \alpha_{12}^{\prime}>0$.

$\left(\mathrm{H}_{4}\right) \beta_{12} / \alpha_{12}>\beta_{12}^{\prime} / \alpha_{12}^{\prime}$.

Lemma 3.4 [13, Lemma 3.2]. Consider the following algebraic equations:

$$
\begin{aligned}
& \bar{a}_{11} N_{1}+\bar{a}_{12} N_{2}+\bar{b}_{1} N_{1} N_{2}=\bar{r}_{1}, \\
& \bar{a}_{21} N_{1}+\bar{a}_{22} N_{2}+\bar{b}_{2} N_{1} N_{2}=\bar{r}_{2} .
\end{aligned}
$$

Assuming that $\left(\mathrm{H}_{1}\right),\left(\mathrm{H}_{2}\right)$ hold, then the following conclusions hold. 
6 Periodic solutions for a discrete plankton model

(i) If $\alpha_{12}>0$, then (3.12) have two positive solutions:

$$
\left(N_{i}\left(\alpha_{12}, \beta_{12}, \gamma_{12}\right), N_{1}\left(\alpha_{21}, \beta_{21}, \gamma_{21}\right)\right), \quad i=1,2 .
$$

(ii) If $\alpha_{21}>0$, then (3.12) have two positive solutions:

$$
\left(N_{1}\left(\alpha_{12}, \beta_{12}, \gamma_{12}\right), N_{i}\left(\alpha_{21}, \beta_{21}, \gamma_{21}\right)\right), \quad i=1,2 .
$$

Lemma 3.5. Assume that $\left(\mathrm{H}_{1}\right)-\left(\mathrm{H}_{3}\right)$ hold, then the following conclusions hold.

(i) $\beta_{12}>0, \beta_{12}^{2}-4 \alpha_{12} \gamma_{12}>0$;

(ii) $\beta_{12}^{\prime}>0, \beta_{12}^{\prime 2}-4 \alpha_{12}^{\prime} \gamma_{12}^{\prime}>0$.

Proof. (i)

$$
\begin{aligned}
& \beta_{12}=\left(\frac{\bar{b}_{1}}{\bar{a}_{11}}+\frac{\bar{a}_{12}}{\bar{r}_{1}}\right) \gamma_{21}+\left(\frac{\bar{r}_{1} \alpha_{12}}{\bar{a}_{11}}+\frac{\bar{a}_{11} \gamma_{12}}{\bar{r}_{1}}\right)>0, \\
& \beta_{12}^{2}-4 \alpha_{12} \gamma_{12}=\left(\frac{\bar{b}_{1}}{\bar{a}_{11}}+\frac{\bar{a}_{12}}{\bar{r}_{1}}\right)^{2} \gamma_{21}^{2}+\left(\frac{\bar{r}_{1} \alpha_{12}}{\bar{a}_{11}}-\frac{\bar{a}_{11} \gamma_{12}}{\bar{r}_{1}}\right)^{2} \\
&+2\left(\frac{\bar{b}_{1}}{\bar{a}_{11}}+\frac{\bar{a}_{12}}{\bar{r}_{1}}\right)\left(\frac{\bar{r}_{1} \alpha_{12}}{\bar{a}_{11}}+\frac{\bar{a}_{11} \gamma_{12}}{\bar{r}_{1}}\right) \gamma_{21}>0 .
\end{aligned}
$$

(ii)

$$
\begin{aligned}
\beta_{12}^{\prime}=\left(\frac{\bar{b}_{1}}{\bar{a}_{11}}+\right. & \left.\frac{\bar{a}_{12}}{\bar{r}_{1}}\right) \gamma_{21}^{\prime \prime}+\left(\frac{\bar{r}_{1} \alpha_{12}^{\prime} e^{\bar{R}_{1} \omega}}{\bar{a}_{11}}+\frac{\bar{a}_{11} \gamma_{12}^{\prime}}{\bar{r}_{1} e^{\bar{R}_{1} \omega}}\right)>0, \\
\beta_{12}^{\prime 2}-4 \alpha_{12}^{\prime} \gamma_{12}^{\prime}= & \left(\frac{\bar{b}_{1}}{\bar{a}_{11}}+\frac{\bar{a}_{12}}{\bar{r}_{1}}\right)^{2} \gamma_{21}^{\prime \prime 2}+\left(\frac{\bar{r}_{1} \alpha_{12}^{\prime} e^{\bar{R}_{1} \omega}}{\bar{a}_{11}}-\frac{\bar{a}_{11} \gamma_{12}^{\prime}}{\bar{r}_{1} e^{\bar{R}_{1} \omega}}\right)^{2} \\
& +2\left(\frac{\bar{b}_{1}}{\bar{a}_{11}}+\frac{\bar{a}_{12}}{\bar{r}_{1}}\right)\left(\frac{\bar{r}_{1} \alpha_{12}^{\prime} e^{\bar{R}_{1} \omega}}{\bar{a}_{11}}+\frac{\bar{a}_{11} \gamma_{12}^{\prime}}{\bar{r}_{1} e^{\bar{R}_{1} \omega}}\right) \gamma_{21}^{\prime \prime}>0 .
\end{aligned}
$$

Lemma 3.6. Assume that $\left(\mathrm{H}_{1}\right)-\left(\mathrm{H}_{4}\right)$ hold, then the following conclusions hold,

$$
\begin{aligned}
& N_{1}\left(\alpha_{12}, \beta_{12}+m, \gamma_{12}-n\right)<N_{1}\left(\alpha_{12}, \beta_{12}, \gamma_{12}\right)<N_{1}\left(\alpha_{12}^{\prime}, \beta_{12}^{\prime}, \gamma_{12}^{\prime}\right) \\
& \quad<N_{2}\left(\alpha_{12}^{\prime}, \beta_{12}^{\prime}, \gamma_{12}^{\prime}\right)<N_{2}\left(\alpha_{12}, \beta_{12}, \gamma_{12}\right)<N_{2}\left(\alpha_{12}, \beta_{12}+m, \gamma_{12}-n\right),
\end{aligned}
$$

where

$$
\begin{gathered}
m=\bar{a}_{11} \bar{a}_{22}\left(e^{\bar{R}_{1} \omega}-1\right)+\bar{b}_{1} \bar{r}_{2}\left(e^{\left(\bar{R}_{1}+\bar{R}_{2}\right) \omega}-1\right)>0, \\
n=\bar{a}_{12} \bar{r}_{2}\left(e^{\bar{R}_{2} \omega}-1\right)>0 .
\end{gathered}
$$

Proof. Under the conditions that $\alpha>0, \beta>0, \gamma>0, \beta^{2}-4 \alpha \gamma>0$, we have

$$
\begin{aligned}
& N_{1}(\alpha, \beta, \gamma)=\frac{2 \gamma}{\beta+\sqrt{\beta^{2}-4 \alpha \gamma}}=\frac{2 \gamma / \alpha}{\beta / \alpha+\sqrt{\beta^{2} / \alpha^{2}-4(\gamma / \alpha)}}=N_{1}\left(1, \frac{\beta}{\alpha}, \frac{\gamma}{\alpha}\right), \\
& N_{2}(\alpha, \beta, \gamma)=\frac{\beta+\sqrt{\beta^{2}-4 \alpha \gamma}}{2 \alpha}=\frac{1}{2}\left(\frac{\beta}{\alpha}+\sqrt{\frac{\beta^{2}}{\alpha^{2}}-4 \frac{\gamma}{\alpha}}\right)=N_{2}\left(1, \frac{\beta}{\alpha}, \frac{\gamma}{\alpha}\right) .
\end{aligned}
$$


Thus $N_{1}(\alpha, \beta, \gamma)\left(N_{2}(\alpha, \beta, \gamma)\right)$ is increasing (decreasing) in the first variable, decreasing (increasing) in the second variable, increasing (decreasing) in the third variable. Notice that

$$
\alpha_{12}^{\prime \prime}>\alpha_{12}>\alpha_{12}^{\prime}>0, \quad \gamma_{12}^{\prime}>\gamma_{12}>\gamma_{12}^{\prime \prime}>0
$$

we have

$$
\frac{\gamma_{12}^{\prime}}{\alpha_{12}^{\prime}}>\frac{\gamma_{12}}{\alpha_{12}}
$$

So from (3.19), (3.20), (3.21) and $\left(\mathrm{H}_{1}\right)-\left(\mathrm{H}_{4}\right)$, we obtain that

$$
\begin{gathered}
N_{1}\left(\alpha_{12}, \beta_{12}+m, \gamma_{12}-n\right)<N_{1}\left(\alpha_{12}, \beta_{12}, \gamma_{12}\right)=N_{1}\left(1, \frac{\beta_{12}}{\alpha_{12}}, \frac{\gamma_{12}}{\alpha_{12}}\right) \\
<N_{1}\left(1, \frac{\beta_{12}^{\prime}}{\alpha_{12}^{\prime}}, \frac{\gamma_{12}^{\prime}}{\alpha_{12}^{\prime}}\right)=N_{1}\left(\alpha_{12}^{\prime}, \beta_{12}^{\prime}, \gamma_{12}^{\prime}\right)<N_{2}\left(\alpha_{12}^{\prime}, \beta_{12}^{\prime}, \gamma_{12}^{\prime}\right)=N_{2}\left(1, \frac{\beta_{12}^{\prime}}{\alpha_{12}^{\prime}}, \frac{\gamma_{12}^{\prime}}{\alpha_{12}^{\prime}}\right) \\
<N_{2}\left(1, \frac{\beta_{12}}{\alpha_{12}}, \frac{\gamma_{12}}{\alpha_{12}}\right)=N_{2}\left(\alpha_{12}, \beta_{12}, \gamma_{12}\right)<N_{2}\left(\alpha_{12}, \beta_{12}+m, \gamma_{12}-n\right) .
\end{gathered}
$$

THEOREM 3.7. In addition to $\left(\mathrm{H}_{1}\right)-\left(\mathrm{H}_{3}\right)$, assume further that system (2.3) satisfies

$\left(\mathrm{H}_{5}\right) N_{1}\left(\alpha_{12}, \beta_{12}, \gamma_{12}\right)<N_{1}\left(\alpha_{12}^{\prime}, \beta_{12}^{\prime}, \gamma_{12}^{\prime}\right)<N_{2}\left(\alpha_{12}, \beta_{12}, \gamma_{12}\right)$.

Then system (2.3) has at least two positive $\omega$-periodic solutions.

Proof. Since we are concerned with positive solutions of (2.3), we make the change of variables,

$$
N_{i}(k)=\exp \left(x_{i}(k)\right), \quad i=1,2 .
$$

Then (2.3) is rewritten as

$$
\begin{aligned}
x_{i}(k+1)-x_{i}(k)= & r_{i}(k)-a_{i i}(k) \exp \left(x_{i}(k)\right)-a_{i j}(k) \exp \left(x_{j}(k)\right) \\
& -b_{i}(k) \exp \left(x_{i}(k)\right) \exp \left(x_{j}(k)\right),
\end{aligned}
$$

where $i, j=1,2, i \neq j$. Take $X=Y=l^{\omega},(L x)(k)=x(k+1)-x(k)$, and denote

$(\mathcal{N} x)(k)=\left(\begin{array}{l}r_{1}(k)-a_{11}(k) \exp \left(x_{1}(k)\right)-a_{12}(k) \exp \left(x_{2}(k)\right)-b_{1}(k) \exp \left(x_{1}(k)\right) \exp \left(x_{2}(k)\right) \\ r_{2}(k)-a_{22}(k) \exp \left(x_{2}(k)\right)-a_{21}(k) \exp \left(x_{1}(k)\right)-b_{2}(k) \exp \left(x_{2}(k)\right) \exp \left(x_{1}(k)\right)\end{array}\right)$,

for any $x \in X$ and $k \in Z$. It follows from Lemma 3.2 that $L$ is a bounded linear operator and

$$
\operatorname{ker} L=l_{c}^{\omega}, \quad \operatorname{Im} L=l_{0}^{\omega}, \quad \operatorname{dim} \operatorname{ker} L=2=\operatorname{codim} \operatorname{Im} L,
$$

then it follows that $L$ is a Fredholm mapping of index zero. 
8 Periodic solutions for a discrete plankton model

Define

$$
P x=\frac{1}{\omega} \sum_{s=0}^{\omega-1} x(s), \quad x \in X, \quad Q y=\frac{1}{\omega} \sum_{s=0}^{\omega-1} y(s), \quad y \in Y .
$$

It is not difficult to show that $P$ and $Q$ are two continuous projectors such that

$$
\operatorname{Im} P=\operatorname{ker} L, \quad \operatorname{Im} L=\operatorname{ker} Q=\operatorname{Im}(I-Q) .
$$

Furthermore, the generalized inverse (of $L) K_{p}: \operatorname{Im} L \rightarrow \operatorname{ker} P \cap \operatorname{Dom} L$ exists and is given by

$$
K_{p}(z)=\sum_{s=0}^{k-1} z(s)-\frac{1}{\omega} \sum_{s=0}^{\omega-1}(\omega-s) z(s) .
$$

Notice that $Q \mathcal{N}, K_{p}(I-Q) \mathcal{N}$ are continuous and $X$ is a finite-dimensional Banach space, it is not difficult to show that $\overline{K_{p}(I-Q) \mathcal{N}(\bar{\Omega})}$ is compact for any open bounded set $\Omega \subset X$. Moreover, $Q \mathcal{N}(\bar{\Omega})$ is bounded. Thus, $\mathcal{N}$ is $L$-compact on with any open bounded set $\Omega \subset X$.

Corresponding to the operator equation $L x=\lambda \mathcal{N} x, \lambda \in(0,1)$, we have

$$
\begin{gathered}
x_{i}(k+1)-x_{i}(k)=\lambda\left[r_{i}(k)-a_{i i}(k) \exp \left(x_{i}(k)\right)-a_{i j}(k) \exp \left(x_{j}(k)\right)\right. \\
\left.-b_{i}(k) \exp \left(x_{i}(k)\right) \exp \left(x_{j}(k)\right)\right],
\end{gathered}
$$

where $i, j=1,2, i \neq j$. Suppose that $x=\left(x_{1}(k), x_{2}(k)\right)^{T} \in X$ is a solution of (3.30) for a certain $\lambda \in(0,1)$. Summing on both sides of (3.30) from 0 to $\omega-1$ about $k$, we get

$$
\begin{aligned}
0 & =\sum_{k=0}^{\omega-1}\left(x_{i}(k+1)-x_{i}(k)\right) \\
& =\lambda \sum_{k=0}^{\omega-1}\left[r_{i}(k)-a_{i i}(k) \exp \left(x_{i}(k)\right)-a_{i j}(k) \exp \left(x_{j}(k)\right)-b_{i}(k) \exp \left(x_{i}(k)\right) \exp \left(x_{j}(k)\right)\right],
\end{aligned}
$$

that is

$$
\bar{r}_{i} \omega=\sum_{k=0}^{\omega-1}\left[a_{i i}(k) \exp \left(x_{i}(k)\right)+a_{i j}(k) \exp \left(x_{j}(k)\right)+b_{i}(k) \exp \left(x_{i}(k)\right) \exp \left(x_{j}(k)\right)\right],
$$

where $i, j=1,2, i \neq j$.

It follows from (3.30) that

$$
x_{i}(k+1)-x_{i}(k)<\left|r_{i}(k)\right|, \quad k \in Z, i=1,2 .
$$

Since $x(t) \in X$, there exist $\xi_{i}, \eta_{i} \in I_{\omega}$ such that

$$
x_{i}\left(\xi_{i}\right)=\min _{k \in I_{\omega}}\left\{x_{i}(k)\right\}, \quad x_{i}\left(\eta_{i}\right)=\max _{k \in I_{\omega}}\left\{x_{i}(k)\right\}, \quad i=1,2 .
$$


From (3.32), (3.34), one obtains

$$
\begin{aligned}
& \bar{a}_{11} \exp \left(x_{1}\left(\eta_{1}\right)\right)+\bar{a}_{12} \exp \left(x_{2}\left(\eta_{2}\right)\right)+\bar{b}_{1} \exp \left(x_{1}\left(\eta_{1}\right)\right) \exp \left(x_{2}\left(\eta_{2}\right)\right) \geq \bar{r}_{1} \\
& \bar{a}_{21} \exp \left(x_{1}\left(\xi_{1}\right)\right)+\bar{a}_{22} \exp \left(x_{2}\left(\xi_{2}\right)\right)+\bar{b}_{2} \exp \left(x_{1}\left(\xi_{1}\right)\right) \exp \left(x_{2}\left(\xi_{2}\right)\right) \leq \bar{r}_{2} .
\end{aligned}
$$

We can derive from Lemma 3.3, (3.33) and (3.36) that

$$
x_{2}\left(\eta_{2}\right) \leq x_{2}\left(\xi_{2}\right)+\bar{R}_{2} \omega \leq \ln \frac{\bar{r}_{2}-\bar{a}_{21} \exp \left(x_{1}\left(\xi_{1}\right)\right)}{\bar{a}_{22}+\bar{b}_{2} \exp \left(x_{1}\left(\xi_{1}\right)\right)}+\bar{R}_{2} \omega,
$$

which, together with (3.35), leads to

$$
\begin{aligned}
\exp \left(x_{1}\left(\eta_{1}\right)\right) & \geq \frac{\bar{r}_{1}-\bar{a}_{12} \exp \left(x_{2}\left(\eta_{2}\right)\right)}{\bar{a}_{11}+\bar{b}_{1} \exp \left(x_{2}\left(\eta_{2}\right)\right)} \\
& \geq \frac{\bar{r}_{1}\left(\bar{a}_{22}+\bar{b}_{2} \exp \left(x_{1}\left(\xi_{1}\right)\right)\right)-\bar{a}_{12} \exp \left(\bar{R}_{2} \omega\right)\left(\bar{r}_{2}-\bar{a}_{21} \exp \left(x_{1}\left(\xi_{1}\right)\right)\right)}{\bar{a}_{11}\left(\bar{a}_{22}+\bar{b}_{2} \exp \left(x_{1}\left(\xi_{1}\right)\right)\right)+\bar{b}_{1} \exp \left(\bar{R}_{2} \omega\right)\left(\bar{r}_{2}-\bar{a}_{21} \exp \left(x_{1}\left(\xi_{1}\right)\right)\right)} .
\end{aligned}
$$

From Lemma 3.3 and (3.33), we have

$$
x_{1}\left(\xi_{1}\right)>x_{1}\left(\eta_{1}\right)-\bar{R}_{1} \omega
$$

This is

$$
\exp \left(x_{1}\left(\xi_{1}\right)\right)>\exp \left(x_{1}\left(\eta_{1}\right)\right) \exp \left(-\bar{R}_{1} \omega\right),
$$

which, together with (3.38), leads to

$$
\begin{aligned}
\exp \left(\bar{R}_{1} \omega\right) \exp \left(x_{1}\left(\xi_{1}\right)\right) & \\
> & \frac{\bar{r}_{1}\left(\bar{a}_{22}+\bar{b}_{2} \exp \left(x_{1}\left(\xi_{1}\right)\right)\right)-\bar{a}_{12} \exp \left(\bar{R}_{2} \omega\right)\left(\bar{r}_{2}-\bar{a}_{21} \exp \left(x_{1}\left(\xi_{1}\right)\right)\right)}{\bar{a}_{11}\left(\bar{a}_{22}+\bar{b}_{2} \exp \left(x_{1}\left(\xi_{1}\right)\right)\right)+\bar{b}_{1} \exp \left(\bar{R}_{2} \omega\right)\left(\bar{r}_{2}-\bar{a}_{21} \exp \left(x_{1}\left(\xi_{1}\right)\right)\right)},
\end{aligned}
$$

which implies

$$
\alpha_{12}^{\prime \prime} \exp \left(2 x_{1}\left(\xi_{1}\right)\right)-\beta_{12}^{\prime \prime} \exp \left(x_{1}\left(\xi_{1}\right)\right)+\gamma_{12}^{\prime \prime}<0 .
$$

So from (3.20), one obtains

$$
\alpha_{12} \exp \left(2 x_{1}\left(\xi_{1}\right)\right)-\left(\beta_{12}+m\right) \exp \left(x_{1}\left(\xi_{1}\right)\right)+\gamma_{12}-n<0,
$$

where

$$
m=\bar{a}_{11} \bar{a}_{22}\left(e^{\bar{R}_{1} \omega}-1\right)+\bar{b}_{1} \bar{r}_{2}\left(e^{\left(\bar{R}_{1}+\bar{R}_{2}\right) \omega}-1\right)>0, \quad n=\bar{a}_{11} \bar{r}_{2}\left(e^{\bar{R}_{2} \omega}-1\right)>0 .
$$

According to (i) of Lemma 3.5, we obtain

$$
\left(\beta_{12}+m\right)^{2}-4 \alpha_{12}\left(\gamma_{12}-n\right)>\beta_{12}^{2}-4 \alpha_{12} \gamma_{12}>0 .
$$


10 Periodic solutions for a discrete plankton model

Therefore, the equation

$$
\alpha_{12} x^{2}-\left(\beta_{12}+m\right) x+\gamma_{12}-n=0
$$

has two positive solutions

$$
N_{i}\left(\alpha_{12}, \beta_{12}+m, \gamma_{12}-n\right), \quad i=1,2
$$

Thus, we have

$$
N_{1}\left(\alpha_{12}, \beta_{12}+m, \gamma_{12}-n\right)<\exp \left(x_{1}\left(\xi_{1}\right)\right)<N_{2}\left(\alpha_{12}, \beta_{12}+m, \gamma_{12}-n\right) .
$$

In a similar way as the above proof, we can conclude from

$$
\begin{aligned}
& \bar{a}_{21} \exp \left(x_{1}\left(\eta_{1}\right)\right)+\bar{a}_{22} \exp \left(x_{2}\left(\eta_{2}\right)\right)+\bar{b}_{2} \exp \left(x_{1}\left(\eta_{1}\right)\right) \exp \left(x_{2}\left(\eta_{2}\right)\right) \geq \bar{r}_{2}, \\
& \bar{a}_{11} \exp \left(x_{1}\left(\xi_{1}\right)\right)+\bar{a}_{12} \exp \left(x_{2}\left(\xi_{2}\right)\right)+\bar{b}_{1} \exp \left(x_{1}\left(\xi_{1}\right)\right) \exp \left(x_{2}\left(\xi_{2}\right)\right) \leq \bar{r}_{1},
\end{aligned}
$$

that

$$
\alpha_{12}^{\prime} \exp \left(2 x_{1}\left(\eta_{1}\right)\right)-\beta_{12}^{\prime} \exp \left(x_{1}\left(\eta_{1}\right)\right)+\gamma_{12}^{\prime}>0 .
$$

According to (ii) of Lemma 3.5, one has

$$
\beta_{12}^{\prime 2}-4 \alpha_{12}^{\prime} \gamma_{12}^{\prime}>0
$$

Therefore, the equation

$$
\alpha_{12}^{\prime} x^{2}-\beta_{12}^{\prime} x+\gamma_{12}^{\prime}=0
$$

has two positive solutions

$$
N_{i}\left(\alpha_{12}^{\prime}, \beta_{12}^{\prime}, \gamma_{12}^{\prime}\right), \quad i=1,2
$$

Thus, we have

$$
\exp \left(x_{1}\left(\eta_{1}\right)\right)>N_{2}\left(\alpha_{12}^{\prime}, \beta_{12}^{\prime}, \gamma_{12}^{\prime}\right), \quad \text { or } \quad \exp \left(x_{1}\left(\eta_{1}\right)\right)<N_{1}\left(\alpha_{12}^{\prime}, \beta_{12}^{\prime}, \gamma_{12}^{\prime}\right)
$$

It follows from Lemma 3.3, (3.33) and (3.48) that

$$
\begin{aligned}
x_{1}\left(\eta_{1}\right) & \leq x_{1}\left(\xi_{1}\right)+\bar{R}_{1} \omega \\
& <\ln N_{2}\left(\alpha_{12}, \beta_{12}+m, \gamma_{12}-n\right)+\bar{R}_{1} \omega:=H .
\end{aligned}
$$

On the other hand, it follows from (3.32) and (3.34) that

$$
\bar{a}_{i i} \omega \exp \left(x_{i}\left(\xi_{i}\right)\right) \leq \sum_{k=0}^{\omega-1} a_{i i}(k) \exp \left(x_{i}(k)\right)<\bar{r}_{i} \omega, \quad i=1,2,
$$


that is

$$
x_{i}\left(\xi_{i}\right)<\ln \frac{\bar{r}_{i}}{\bar{a}_{i i}}, \quad i=1,2 .
$$

From Lemma 3.3, (3.33) and (3.57), one obtains

$$
x_{i}(k) \leq x_{i}\left(\xi_{i}\right)+\bar{R}_{i} \omega<\ln \frac{\bar{r}_{i}}{\bar{a}_{i i}}+\bar{R}_{i} \omega, \quad k \in Z, i=1,2 .
$$

It follows from (3.32) and (3.34) that

$$
\begin{aligned}
\bar{r}_{2} \omega & =\sum_{k=0}^{\omega-1}\left[a_{22}(k) \exp \left(x_{2}(k)\right)+a_{21}(k) \exp \left(x_{1}(k)\right)+b_{2}(k) \exp \left(x_{2}(k)\right) \exp \left(x_{1}(k)\right)\right] \\
& \leq \sum_{j=1}^{2} \bar{a}_{2 j} \omega \exp \left(x_{j}\left(\eta_{j}\right)\right)+\bar{b}_{2} \omega \exp \left(x_{1}\left(\eta_{1}\right)\right) \exp \left(x_{2}\left(\eta_{2}\right)\right),
\end{aligned}
$$

which implies that

$$
\exp \left(x_{2}\left(\eta_{2}\right)\right) \geq \frac{\bar{r}_{2}-\bar{a}_{21} \exp \left(x_{1}\left(\eta_{1}\right)\right)}{\bar{a}_{22}+\bar{b}_{2} \exp \left(x_{1}\left(\eta_{1}\right)\right)} .
$$

From (3.58) and (3.60), we have

$$
x_{2}\left(\eta_{2}\right) \geq \ln \frac{\bar{a}_{11} \bar{r}_{2}-\bar{a}_{21} \bar{r}_{1} \exp \left(\bar{R}_{1} \omega\right)}{\bar{a}_{11} \bar{a}_{22}+\bar{b}_{2} \bar{r}_{1} \exp \left(\bar{R}_{1} \omega\right)}:=M,
$$

which, together with Lemma 3.3, leads to

$$
x_{2}(k) \geq x_{2}\left(\eta_{2}\right)-\bar{R}_{2} \omega>M-\bar{R}_{2} \omega .
$$

By (3.58) and (3.62), we obtain that

$$
\left|x_{2}(k)\right|<\max \left\{\left|\ln \frac{\bar{r}_{2}}{\bar{a}_{22}}+\bar{R}_{2} \omega\right|,\left|M-\bar{R}_{2} \omega\right|\right\}:=A, \quad k \in Z .
$$

Now, let us consider $Q \mathcal{N} x$ with $x=\left(x_{1}, x_{2}\right) \in R^{2}$. Note that

$$
Q \mathcal{N}\left(x_{1}, x_{2}\right)=\left(\begin{array}{l}
\bar{r}_{1}-\bar{a}_{11} \exp \left(x_{1}\right)-\bar{a}_{12} \exp \left(x_{2}\right)-\bar{b}_{1} \exp \left(x_{1}\right) \exp \left(x_{2}\right) \\
\bar{r}_{2}-\bar{a}_{21} \exp \left(x_{1}\right)-\bar{a}_{22} \exp \left(x_{2}\right)-\bar{b}_{2} \exp \left(x_{1}\right) \exp \left(x_{2}\right)
\end{array}\right) .
$$

According to Lemma 3.4, we can show that $Q \mathcal{N} x=0$ has two distinct solutions

$$
\hat{x}^{i}=\left(\ln N_{i}\left(\alpha_{12}, \beta_{12}, \gamma_{12}\right), \ln N_{1}\left(\alpha_{21}, \beta_{21}, \gamma_{21}\right)\right), \quad i=1,2 .
$$

Choose $C>0$ such that

$$
C>\left|\ln N_{1}\left(\alpha_{21}, \beta_{21}, \gamma_{21}\right)\right|
$$


Let

$$
\begin{aligned}
& \Omega_{1}=\left\{x \in X \mid \begin{array}{l}
x_{1}(k) \in\left(\ln N_{1}\left(\alpha_{12}, \beta_{12}+m, \gamma_{12}-n\right), \ln N_{1}\left(\alpha_{12}^{\prime}, \beta_{12}^{\prime}, \gamma_{12}^{\prime}\right)\right), \\
\left|x_{2}(k)\right|<A+C .
\end{array}\right\}, \\
& \Omega_{2}=\left\{\begin{array}{l}
x \in X \mid \begin{array}{l}
\min _{k \in I_{\omega}} x_{1}(k) \in\left(\ln N_{1}\left(\alpha_{12}, \beta_{12}+m, \gamma_{12}-n\right), \ln N_{2}\left(\alpha_{12}, \beta_{12}+m, \gamma_{12}-n\right),\right. \\
\max _{k \in I_{\omega}} x_{1}(k) \in\left(\min \left\{\ln N_{2}\left(\alpha_{12}, \beta_{12}, \gamma_{12}\right), \ln N_{2}\left(\alpha_{12}^{\prime}, \beta_{12}^{\prime}, \gamma_{12}^{\prime}\right)\right\}-\delta, H\right), \\
x_{2}(k) \mid<A+C .
\end{array}
\end{array}\right\},
\end{aligned}
$$

where $\delta$ is a constant such that

$$
\min \left\{\ln N_{2}\left(\alpha_{12}, \beta_{12}, \gamma_{12}\right), \ln N_{2}\left(\alpha_{12}^{\prime}, \beta_{12}^{\prime}, \gamma_{12}^{\prime}\right)\right\}-\ln N_{1}\left(\alpha_{12}^{\prime}, \beta_{12}^{\prime}, \gamma_{12}^{\prime}\right)>\delta>0
$$

Then both $\Omega_{1}$ and $\Omega_{2}$ are bounded open subsets of $X$. It follows from Lemma 3.4 and (3.66) that $\hat{x}^{i} \in \Omega_{i}, i=1,2$. With the help of (3.48), (3.54), (3.55), (3.63) and $\left(\mathrm{H}_{5}\right)$, it is easy to see that $\bar{\Omega}_{1} \cap \bar{\Omega}_{2}=\phi$ and $\Omega_{i}$ satisfies the requirement (a) in Lemma 3.1 for $i=1,2$. Moreover, $Q \mathcal{N} x \neq 0$ for $x \in \partial \Omega_{i} \cap \operatorname{Ker} L, i=1,2$. A direct computation gives

$$
\operatorname{deg}_{B}\left(J Q \mathcal{N}, \Omega_{i} \cap \operatorname{Ker} L, 0\right) \neq 0 .
$$

Here $J$ is taken as the identity mapping $\operatorname{since} \operatorname{Im} Q=\operatorname{Ker} L$. So far we have proved that $\Omega_{i}$ satisfies all the assumptions in Lemma 3.1. Hence (3.24) has at least two $\omega$-periodic solutions $\breve{x}^{i}$ with $\breve{x}^{i} \in \operatorname{Dom} L \cap \bar{\Omega}_{i}(i=1,2)$. Obviously $\breve{x}^{i}(i=1,2)$ are different. Let $\breve{N}_{j}^{i}(k)=$ $\exp \left(\breve{x}_{j}^{i}(k)\right), i, j=1,2$. Then $\breve{N}^{i}=\left(\breve{N}_{1}^{i}, \breve{N}_{2}^{i}\right)(i=1,2)$ are two different positive $\omega$-periodic solutions of (2.3). The proof is complete.

With the help of Lemma 3.6 and Theorem 3.7, we have the following.

Corollary 3.8. Under Assumptions $\left(\mathrm{H}_{1}\right)-\left(\mathrm{H}_{4}\right)$, system (2.3) has at least two positive wperiodic solutions.

Example 3.9. As an application of Corollary 3.8, we consider the following system

$$
\begin{aligned}
N_{1}(k+1)=N_{1}(k) \exp \{0 & 0002+0.0002 \cos (\pi k / 50) \\
- & (0.0001+0.00005 \cos (\pi k / 50)) N_{1}(k) \\
- & (1000+\cos (\pi k / 50)) N_{2}(k) \\
& \left.-(20000+\cos (\pi k / 50)) N_{1}(k) N_{2}(k)\right\}, \\
N_{2}(k+1)=N_{1}(k) \exp \{0 & 00041+0.00041 \cos (\pi k / 50) \\
- & (0.0002+0.0001 \cos (\pi k / 50)) N_{1}(k) \\
- & (10000+\cos (\pi k / 50)) N_{2}(k) \\
- & \left.(20000+\cos (\pi k / 50)) N_{1}(k) N_{2}(k)\right\} .
\end{aligned}
$$


A direct computation gives that

$$
\begin{gathered}
\bar{r}_{1}=0.0002=\bar{R}_{1}, \quad \bar{r}_{2}=0.00041=\bar{R}_{2}, \quad \bar{a}_{11}=0.0001, \quad \bar{a}_{12}=1000, \\
\bar{a}_{21}=0.0002, \quad \bar{a}_{22}=10000, \quad \bar{b}_{1}=\bar{b}_{2}=20000, \quad \omega=100, \\
\alpha_{12}^{\prime}>1.91629, \quad \gamma_{12}^{\prime \prime}>1.57284, \quad \gamma_{21}^{\prime \prime}>1.9194 \times 10^{-10}, \\
\alpha_{12}^{\prime} \beta_{12}-\alpha_{12} \beta_{12}^{\prime}>0.00904 .
\end{gathered}
$$

So according to Corollary 3.8, the above system has at least two positive 100-periodic solutions.

Similar to the proof of Theorem 3.7, we can prove the following results.

THEOREM 3.10. In addition to $\left(\mathrm{H}_{1}\right)$ and $\left(\mathrm{H}_{2}\right)$, assume further that system (2.3) satisfies $\left(\mathrm{H}_{3}^{\prime}\right) \alpha_{21}^{\prime}>0$.

$\left(\mathrm{H}_{5}^{\prime}\right) N_{1}\left(\alpha_{21}, \beta_{21}, \gamma_{21}\right)<N_{1}\left(\alpha_{21}^{\prime}, \beta_{21}^{\prime}, \gamma_{21}^{\prime}\right)<N_{2}\left(\alpha_{21}, \beta_{21}, \gamma_{21}\right)$.

Then system (2.3) has at least two positive $\omega$-periodic solutions.

Corollary 3.11. In addition to $\left(\mathrm{H}_{1}\right),\left(\mathrm{H}_{2}\right)$ and $\left(\mathrm{H}_{3}^{\prime}\right)$, assume further that system (2.3) satisfies

$\left(\mathrm{H}_{4}^{\prime}\right) \beta_{21} / \alpha_{21}>\beta_{21}^{\prime} / \alpha_{21}^{\prime}$.

Then system (2.3) has at least two positive $\omega$-periodic solutions.

\section{Acknowledgment}

This research is supported by the National Natural Science Foundation of China (No. 10161007, 10561004).

\section{References}

[1] R. Arditi, L. R. Ginzburg, and H. R. Akcakaya, Variation in plankton densities among lakes: a case for ratio-dependent predation models, The American Naturalist 138 (1991), 1287-1296.

[2] J. Chattopadhyay, Effect of toxic substances on a two-species competitive system, Ecological Modelling 84 (1996), no. 1-3, 287-289.

[3] Y. Chen, Multiple periodic solutions of delayed predator-prey systems with type IV functional responses, Nonlinear Analysis: Real World Applications 5 (2004), no. 1, 45-53.

[4] M. Fan and K. Wang, Periodic solutions of a discrete time nonautonomous ratio-dependent predator-prey system, Mathematical and Computer Modelling 35 (2002), no. 9-10, 951-961.

[5] H. I. Freedman and J. Wu, Periodic solutions of single-species models with periodic delay, SIAM Journal on Mathematical Analysis 23 (1992), no. 3, 689-701.

[6] R. E. Gaines and J. L. Mawhin, Coincidence Degree and Nonlinear Differential Equations, Lecture Notes in Mathematics, vol. 568, Springer, Berlin, 1977.

[7] J. A. Hellebust, Extracellular Products in Algal Physiology and Biochemistry, edited by W. D. P. Stewart, University of California Press, California, 1974.

[8] Y. Kuang, Delay Differential Equations with Applications in Population Dynamics, Mathematics in Science and Engineering, vol. 191, Academic Press, Massachusetts, 1993.

[9] J. Maynard-Smith, Models in Ecology, Cambridge University Press, Cambridge, UK, 1974.

[10] A. Mukhopadhyay, J. Chattopadhyay, and P. K. Tapaswi, A delay differential equations model of plankton allelopathy, Mathematical Biosciences 149 (1998), no. 2, 167-189.

[11] E. L. Rice, Allelopathy, Academic Press, New York, 1984. 


\section{Periodic solutions for a discrete plankton model}

[12] R. Y. Zhang, Z. C. Wang, Y. Chen, and J. Wu, Periodic solutions of a single species discrete population model with periodic harvest/stock, Computers \& Mathematics with Applications 39 (2000), no. 1-2, 77-90.

[13] J. Zhen and Z. Ma, Periodic solutions for delay differential equations model of plankton allelopathy, Computers \& Mathematics with Applications 44 (2002), no. 3-4, 491-500.

Jianbao Zhang: Center for Nonlinear Science Studies, Kunming University of Science and Technology, Kunming, Yunnan 650093, China

E-mail address: jianbaozhang@163.com

Hui Fang: Center for Nonlinear Science Studies, Kunming University of Science and Technology, Kunming, Yunnan 650093, China

E-mail address: huifang@public.km.yn.cn 